\title{
NONCONSTANT SUM RED-AND-BLACK \\ GAMES WITH BET-DEPENDENT WIN PROBABILITY FUNCTION
}

\author{
LAURA PONTIGGIA, ${ }^{*}$ University of the Sciences in Philadelphia
}

\begin{abstract}
In this paper we investigate a class of $N$-person nonconstant sum red-and-black games with bet-dependent win probability functions. We assume that $N$ players and a gambling house are engaged in a game played in stages, where the player's probability of winning at each stage is a function $f$ of the ratio of his bet to the sum of all the players' bets. However, at each stage of the game there is a positive probability that all the players lose and the gambling house wins their bets. We prove that if the win probability function is super-additive and it satisfies $f(s) f(t) \leq f(s t)$, then a bold strategy is optimal for all players.
\end{abstract}

Keywords: Stochastic games; nonconstant sum games; red-and-black

2000 Mathematics Subject Classification: Primary 91A15

Secondary 91A05; 91A06

\section{Introduction}

One of the most interesting examples in Dubins and Savage [2] is the famous red-and-black gambling problem. In the discrete version this gambling problem can be described as follows. A player has an initial nonnegative integer fortune $x$ and wants to reach a goal of $g$ units by betting at each stage of the game an integral amount $s$ not greater than his current fortune. If his initial stake is $s \leq x$, his next fortune will be $x+s$ with probability $w$ (with $0<w<1$ ), and $x-s$ with probability $\bar{w}=1-w$. This process continues until the goal $g$ is reached or the player goes broke. The problem for the player is to decide how many units of his current fortune should be staked at each stage in order to maximize his probability of reaching the goal $g$.

Dubins and Savage [2] showed that the solution of this problem depends on the value of the probability $w$. In particular, they showed that in the subfair case (i.e. $w \leq \frac{1}{2}$ ) an optimal strategy is bold play which corresponds to always staking the entire fortune or just what is needed to reach the target, whichever is smaller. In the superfair case (i.e. $w \geq \frac{1}{2}$ ) Ross [5] proved that it is optimal for the player to play timidly, that is, always to stake 1 unit of his current fortune when he is between 1 and $g-1$ and 0 otherwise. A gambling theoretical proof of Ross's result can be found in Maitra and Sudderth [3].

Pontiggia [4] introduced two-person red-and-black games, in which two players hold an integral amount of money and they both aim to win the other player's fortune. Chen and Hsiau [1] extended the results in Pontiggia [4] to two-person red-and-black games with betdependent win probability functions. At the present time we are not aware of any nontrivial results establishing Nash equilibria for $N$-person red-and-black games.

Received 30 January 2007; revision received 6 March 2007.

* Postal address: Department of Mathematics, Physics and Computer Science, University of the Sciences in Philadelphia, 600 South 43rd Street, Philadelphia, PA 19104, USA. Email address: 1.pontig@usip.edu 
This paper considers an $N$-person nonconstant sum red-and-black game, in which we have $N$ players and a gambling house that at each stage of the game has a positive probability of winning the players' bets.

In Section 2 we introduce the notation and the terminology we will use in the following sections and set up the formulation of the nonconstant sum red-and-black game.

In Section 3, we show that if the win probability function is super-additive (i.e. $\sum_{i=1}^{n} f\left(s_{i}\right) \leq$ $\left.f\left(\sum_{i=1}^{n} s_{i}\right)\right)$ and it satisfies the condition $f(s) f(t) \leq f(s t)$, then it is optimal for all players to bet boldly. Several examples are given as applications of this result.

\section{Preliminaries}

We imagine there are $N$ players (with $N \geq 2$ ) who are engaged in a game played in stages, where they all aim to reach a goal fortune $g$ by betting at each stage an integral amount not greater than their current fortune. However, the gambling house may win some of the players' money.

The initial fortunes of the players form a vector $\left(x_{1}, \ldots, x_{j}, \ldots, x_{N}\right)$, where $x_{j}$ denotes the positive integer fortune held by player $j$, for $j=1, \ldots, N$. Hence, at the initial state, we have a total amount of money in the system equal to $M=\sum_{j=1}^{N} x_{j}$. We assume that $g \leq M<2 g$. During the game the amount of money held by the players can become less than $M$ if, at some stage of the game, all of the players lose and the money goes to the gambling house.

The state space $S$ of this game is defined as the following set of all possible fortunes held by the $N$ players:

$$
S=\left\{\left(x_{1}, \ldots, x_{N}\right): 0 \leq x_{j} \leq g \text { for } j=1, \ldots, N\right\}
$$

This $N$-dimensional state space is discrete and it includes several absorbing states. In fact, after one of the players reaches the goal $g$, or the total amount of money held by the players becomes less than $g$, the game does not move to any other state.

At each stage, each player bids an integral amount of money, which must be less than or equal to the fortune that he holds. We assume that each player chooses his action without any knowledge about the actions chosen by the other players. In fact, all the games we consider are noncooperative games. Suppose that player $j$ bids an amount of money $a_{j}$, where his action set is

$$
A_{j}\left(x_{1}, \ldots, x_{N}\right)= \begin{cases}\left\{1, \ldots, x_{j}\right\} & \text { if } x_{j} \in\{1, \ldots, g-1\} \\ \{0\} & \text { if } x_{j} \in\{0, g\} .\end{cases}
$$

In our game we require each player to bid at least one unit of money if he has a positive fortune less than $g$, i.e. $1 \leq a_{j} \leq x_{j}$ if $x_{j}>0$, for any $j=1, \ldots, N$. If we allow all of them to bid 0 , then for this game there is a trivial, uninteresting Nash equilibrium, in which all players bid 0 forever, and the game stays in the same state perpetually.

The payoff function for each player is given by an indicator function, which takes value 1 if the player reaches the goal $g$, and 0 otherwise. Notice that in this game the total amount of money in the system can stay the same as the original amount $M$ or eventually decrease if, at some stage of the game, the gambling house wins the players' bets. Hence, only one of the players can reach the goal, or if the sum of the players' fortunes becomes less than $g$ none of them will be able to reach it. The sum of the payoffs associated to each set of strategies is not constant (i.e. it can be 0 or 1 ), so the games described here are nonconstant sum stochastic 
games. Notice that at each stage of the game the players have a positive probability of losing at least one unit of money.

\section{Nonconstant sum $N$-person red-and-black game}

Suppose that at stage $m$ each player $j$ has $1 \leq x_{j}^{m} \leq g-1$ units of money, and he bids an amount $1 \leq a_{j}^{m} \leq x_{j}^{m}$, for $j=1, \ldots, N$. The law of motion for the game at stage $m$ is defined by

$$
\begin{aligned}
& \left(x_{1}^{m+1}, \ldots, x_{N}^{m+1}\right) \\
& \quad= \begin{cases}\left(x_{1}^{m}-a_{1}, \ldots, x_{N}^{m}-a_{N}\right) & \text { w.p. } 1-\sum_{j=1}^{N} f\left(\frac{a_{j}}{\sum_{i=1}^{N} a_{i}}\right), \\
\left(x_{1}^{m}+\sum_{i=2}^{N} a_{i}, \ldots, x_{N}^{m}-a_{N}\right) & \text { w.p. } f\left(\frac{a_{1}}{\sum_{i=1}^{N} a_{i}}\right), \\
\vdots & \vdots \\
\left(x_{1}^{m}-a_{1}, \ldots, x_{j}^{m}+\sum_{k \neq j} a_{k}, \ldots, x_{N}^{m}-a_{N}\right) & \text { w.p. } f\left(\frac{a_{j}}{\sum_{i=1}^{N} a_{i}}\right), \\
\vdots & \vdots \\
\left(x_{1}^{m}-a_{1}, \ldots, x_{N}^{m}+\sum_{i=1}^{N-1} a_{i}\right) & \text { w.p. } f\left(\frac{a_{N}}{\sum_{i=1}^{N} a_{i}}\right),\end{cases}
\end{aligned}
$$

where the function $f:[0,1] \rightarrow[0,1]$ is an increasing, continuous nonzero function with $f(0)=0, f(s) \leq s$, which represents the win probability function of the game, and 'w.p.' denotes 'with probability'.

We notice that if $\sum_{j=1}^{N} x_{j}^{m}<g$ at some stage $m$, then the probability of reaching the goal $g$ is equal to zero for all the players; hence, the game stops. Also, after one of the players reaches the goal $g$, he stops staking money; hence, the game stops, and the probability for the other players to reach their goal becomes zero. In conclusion, in our games it is impossible for more than one player to reach the goal because of our assumption that the total amount of money $M$ is smaller than $2 g$.

As $f(s) \leq s$, we see that, for any $j=1, \ldots, N$,

$$
\begin{aligned}
\mathrm{E}\left[x_{j}^{m+1} \mid x_{j}^{m}\right]= & \left(x_{j}^{m}-a_{j}^{m}\right)\left[1-\sum_{k=1}^{N} f\left(\frac{a_{k}^{m}}{\sum_{i=1}^{N} a_{i}^{m}}\right)\right]+\left(x_{j}^{m}+\sum_{k \neq j} a_{k}^{m}\right) f\left(\frac{a_{j}^{m}}{\sum_{i=1}^{N} a_{i}^{m}}\right) \\
& +\left(x_{j}^{m}-a_{j}^{m}\right) \sum_{k \neq j} f\left(\frac{a_{k}^{m}}{\sum_{i=1}^{N} a_{i}^{m}}\right) \\
= & \left(x_{j}^{m}-a_{j}^{m}\right)\left[1-f\left(\frac{a_{j}^{m}}{\sum_{i=1}^{N} a_{i}^{m}}\right)\right]+\left(x_{j}^{m}+\sum_{k \neq j} a_{k}^{m}\right) f\left(\frac{a_{j}^{m}}{\sum_{i=1}^{N} a_{i}^{m}}\right) \\
= & \left(x_{j}^{m}-a_{j}^{m}\right)+f\left(\frac{a_{j}^{m}}{\sum_{i=1}^{N} a_{i}^{m}}\right) \sum_{i=1}^{N} a_{i}^{m} \\
\leq & x_{j}^{m}
\end{aligned}
$$


and

$$
\mathrm{E}\left[x_{j}^{m+1} \mid x_{j}^{m}\right]=x_{j}^{m}
$$

for $x_{j}^{m}=0$ or $g$. Therefore, the processes $\left\{x_{j}^{m}\right\}$, for $j=1, \ldots, N$, of the fortunes of the $N$ players are supermartingales. This means that the game is subfair for all the players.

For a nonconstant sum $N$-person red-and-black game with $1 \leq x_{j} \leq g-1$ and $g \leq \sum_{j=1}^{N} x_{j}<2 g$, for $j=1, \ldots, N$, we can prove the following result.

Theorem 3.1. In a nonconstant sum $N$-person red-and-black game, assume that the win probability function $f$ is super-additive (i.e. $\sum_{i} f\left(s_{i}\right) \leq f\left(\sum_{i} s_{i}\right)$ ) and it satisfies the following condition: $f(s) f(t) \leq f(s t)$. A Nash equilibrium is for all players to play a bold strategy, i.e. $a_{j}\left(x_{1}, \ldots, x_{N}\right)=x_{j}$ for all $j=1, \ldots, N$ and all $\left(x_{1}, \ldots, x_{N}\right) \in S$.

Proof. Suppose that all players adopt a bold strategy and set

$Q_{j}\left(x_{1}, \ldots, x_{N}\right)=\mathrm{P}\left[\right.$ player $j$ reaches his goal $g$ when the game starts at $\left.\left(x_{1}, \ldots, x_{N}\right)\right]$.

The corresponding law of motion at stage $m$ of the game is given by

$$
\left(x_{1}^{m+1}, \ldots, x_{N}^{m+1}\right)= \begin{cases}(0, \ldots, 0) & \text { w.p. } 1-\sum_{j=1}^{N} f\left(\frac{x_{j}}{\sum_{i=1}^{N} x_{i}}\right), \\ \left(\sum_{i=1}^{N} x_{i}, \ldots, 0\right) & \text { w.p. } f\left(\frac{x_{1}}{\sum_{i=1}^{N} x_{i}}\right), \\ \vdots & \vdots \\ \left(0, \ldots, \sum_{i=1}^{N} x_{i}\right) & \text { w.p. } f\left(\frac{x_{N}}{\sum_{i=1}^{N} x_{i}}\right) .\end{cases}
$$

Therefore, the expected return to player $j$ when all $N$ players use a bold strategy is

$$
\begin{aligned}
Q_{j}\left(x_{1}, \ldots, x_{j}, \ldots, x_{N}\right) & =f\left(\frac{x_{j}}{\sum_{i=1}^{N} x_{i}}\right) Q_{j}\left(0, \ldots, \sum_{i=1}^{N} x_{i}, \ldots, 0\right) \\
& =f\left(\frac{x_{j}}{\sum_{i=1}^{N} x_{i}}\right)
\end{aligned}
$$

where

$$
Q_{j}\left(0, \ldots, \sum_{i=1}^{N} x_{i}, \ldots, 0\right)=1
$$

since $\sum_{i=1}^{N} x_{i} \geq g$.

In order to prove that for player $j$ a bold strategy is optimal when all other players play boldly, it suffices to show that $Q_{j}(\cdot)$ is excessive (see Maitra and Sudderth [3, Theorem 3.10]). Hence, we need to show that the expected return that player $j$ gets by initially staking an amount 
$1 \leq a_{j} \leq x_{j}$ and then playing boldly for the rest of the game, is always less than or equal to the expected return he gets playing boldly from the beginning.

Suppose now that the first stage of the game player $j$ stakes an amount $1 \leq a_{j} \leq x_{j}$. His expected return in this case is given by

$$
\begin{aligned}
& \sigma_{j}\left(x_{1}, \ldots, x_{j}, \ldots, x_{N}\right) \\
&= f\left(\frac{x_{1}}{a_{j}+\sum_{i \neq j} x_{i}}\right) Q_{j}\left(a_{j}+\sum_{i \neq j} x_{i}, 0, \ldots, x_{j}-a_{j}, \ldots, 0\right) \\
&+\cdots+f\left(\frac{a_{j}}{a_{j}+\sum_{i \neq j} x_{i}}\right) Q_{j}\left(0, \ldots, \sum_{i=1}^{N} x_{i}, \ldots, 0\right) \\
&+\cdots+f\left(\frac{x_{N}}{a_{j}+\sum_{i \neq j} x_{i}}\right) Q_{j}\left(0, \ldots, x_{j}-a_{j}, \ldots, a_{j}+\sum_{i \neq j} x_{i}\right) \\
&+\left[1-f\left(\frac{a_{j}}{a_{j}+\sum_{i \neq j} x_{i}}\right)-\sum_{i \neq j} f\left(\frac{x_{i}}{a_{j}+\sum_{i \neq j} x_{i}}\right)\right] Q_{j}\left(0, \ldots, x_{j}-a_{j}, \ldots, 0\right),
\end{aligned}
$$

where

$$
\begin{gathered}
Q_{j}\left(0, \ldots, \sum_{i=1}^{N} x_{i}, \ldots, 0\right)=1, \quad \text { since } \sum_{i=1}^{N} x_{i} \geq g, \\
Q_{j}\left(0, \ldots, x_{j}-a_{j}, \ldots, 0\right)=0, \quad \text { since } x_{j}-a_{j}<g, \\
Q_{j}\left(a_{j}+\sum_{i \neq j} x_{i}, 0, \ldots, x_{j}-a_{j}, \ldots, 0\right)=\cdots=Q_{j}\left(0, \ldots, x_{j}-a_{j}, \ldots, a_{j}+\sum_{i \neq j} x_{i}\right) \\
=f\left(\frac{x_{j}-a_{j}}{\sum_{i=1}^{N} x_{i}}\right) .
\end{gathered}
$$

Therefore, we have

$$
\sigma_{j}\left(x_{1}, \ldots, x_{N}\right)=f\left(\frac{a_{j}}{a_{j}+\sum_{i \neq j} x_{i}}\right)+f\left(\frac{x_{j}-a_{j}}{\sum_{i=1}^{N} x_{i}}\right) \sum_{k \neq j} f\left(\frac{x_{k}}{a_{j}+\sum_{i \neq j} x_{i}}\right) .
$$

In order to prove that $Q_{j}(\cdot)$ is excessive we need to show that the following inequality holds:

$$
Q_{j}\left(x_{1}, \ldots, x_{N}\right) \geq \sigma_{j}\left(x_{1}, \ldots, x_{N}\right),
$$

which is equivalent to

$$
f\left(\frac{x_{j}}{\sum_{i=1}^{N} x_{i}}\right) \geq f\left(\frac{a_{j}}{a_{j}+\sum_{i \neq j} x_{i}}\right)+f\left(\frac{x_{j}-a_{j}}{\sum_{i=1}^{N} x_{i}}\right) \sum_{k \neq j} f\left(\frac{x_{k}}{a_{j}+\sum_{i \neq j} x_{i}}\right) .
$$


In order to show that inequality (3.1) holds we need to decompose the quantity $x_{j} /\left(\sum_{i=1}^{N} x_{i}\right)$ as follows:

$$
\begin{aligned}
\frac{x_{j}}{\sum_{i=1}^{N} x_{i}} & =\frac{x_{j}\left(a_{j}+\sum_{k \neq j} x_{k}\right)}{\sum_{i=1}^{N} x_{i}\left(a_{j}+\sum_{k \neq j} x_{k}\right)} \\
& =\frac{x_{j} a_{j}+x_{j} \sum_{k \neq j} x_{k}}{\sum_{i=1}^{N} x_{i}\left(a_{j}+\sum_{k \neq j} x_{k}\right)} \\
& =\frac{a_{j}\left(\sum_{i=1}^{N} x_{i}-\sum_{k \neq j} x_{k}\right)+x_{j} \sum_{k \neq j} x_{k}}{\sum_{i=1}^{N} x_{i}\left(a_{j}+\sum_{k \neq j} x_{k}\right)} \\
& =\frac{a_{j} \sum_{i=1}^{N} x_{i}+\left(x_{j}-a_{j}\right) \sum_{k \neq j} x_{k}}{\sum_{i=1}^{N} x_{i}\left(a_{j}+\sum_{k \neq j} x_{k}\right)} \\
& =\frac{a_{j}}{a_{j}+\sum_{k \neq j} x_{k}}+\frac{\left(x_{j}-a_{j}\right) \sum_{k \neq j} x_{k}}{\left(a_{j}+\sum_{k \neq j} x_{k}\right) \sum_{i=1}^{N} x_{i}} \\
& =\frac{a_{j}}{a_{j}+\sum_{k \neq j} x_{k}}+\frac{x_{j}-a_{j}}{\sum_{i=1}^{N} x_{i}}\left(\sum_{k \neq j} \frac{x_{k}}{a_{j}+\sum_{k \neq j} x_{k}}\right) .
\end{aligned}
$$

Suppose that the win probability function $f$ is super-additive, i.e. $\sum_{i} f\left(s_{i}\right) \leq f\left(\sum_{i} s_{i}\right)$, and it satisfies the condition $f(s) f(t) \leq f(s t)$, for any $s, t \in[0,1]$; hence, we can prove the following result:

$$
\begin{aligned}
f\left(\frac{x_{j}}{\sum_{i=1}^{N} x_{i}}\right) & \geq f\left(\frac{a_{j}}{a_{j}+\sum_{k \neq j} x_{k}}\right)+f\left(\frac{x_{j}-a_{j}}{\sum_{i=1}^{N} x_{i}} \sum_{k \neq j} \frac{x_{k}}{\left(a_{j}+\sum_{k \neq j} x_{k}\right)}\right) \\
& \geq f\left(\frac{a_{j}}{a_{j}+\sum_{k \neq j} x_{k}}\right)+f\left(\frac{x_{j}-a_{j}}{\sum_{i=1}^{N} x_{i}}\right) f\left(\sum_{k \neq j} \frac{x_{k}}{\left(a_{j}+\sum_{k \neq j} x_{k}\right)}\right) \\
& \geq f\left(\frac{a_{j}}{a_{j}+\sum_{k \neq j} x_{k}}\right)+f\left(\frac{x_{j}-a_{j}}{\sum_{i=1}^{N} x_{i}}\right) \sum_{k \neq j} f\left(\frac{x_{k}}{a_{j}+\sum_{k \neq j} x_{k}}\right) .
\end{aligned}
$$

In conclusion, inequality (3.1) holds for any $1 \leq a_{j} \leq x_{j}$. Therefore, we have proved that $Q_{j}(\cdot)$ is excessive for player $j$ and that the profile (Bold, ..., Bold) is a Nash equilibrium for this class of games.

Remarks 3.1. The formulation of this game comes from the idea that $f$ can be viewed as a penalizing function imposed to the players. For example, we can imagine a situation where there is a state agency or the same gambling house that has the power to control the players by penalizing their probability of winning.

Example 3.1. We consider the win probability function $f(s)=w s$, with $0<w<1$, introduced by Pontiggia [4] for the weighted red-and-black game. It is clear that $f(s) \leq s$ for all $s \in(0,1)$ and that this function is super-additive, i.e. $f(s)+f(t)=w s+w t=$ $w(s+t)=f(s+t)$. Also, it is easy to show that this function satisfies the condition $f(s) f(t)=(w s)(w t)=w^{2} s t \leq w s t=f(s t)$. Hence, it follows from Theorem 3.1 that (Bold, ..., Bold) is a Nash equilibrium for a nonconstant sum $N$-person weighted red-andblack game. 
Example 3.2. We consider the win probability function $f(s)=s^{p}$, for some $p \geq 1$, introduced by Chen and Hsiau [1]. It is easy to show that $f(s) \leq s$ and that the function is superadditive, since $f(s)+f(t)=s^{p}+t^{p} \leq(s+t)^{p}=f(s+t)$. Also, the second condition is satisfied since $f(s) f(t)=s^{p} t^{p}=(s t)^{p}=f(s t)$. Hence, it follows from Theorem 3.1 that (Bold, ..., Bold) is a Nash equilibrium for a nonconstant sum $N$-person red-and-black game with this win probability function.

\section{Acknowledgements}

I would like to thank Dr William Sudderth for his suggestions and guidance, and an anonymous referee for his/her very helpful comments and feedback.

\section{References}

[1] Chen, M. And Hsiau, S. (2006). Two-person red-and-black games with bet-dependent win probability functions. J. Appl. Prob. 43, 905-915.

[2] Dubins, L. and Savage, L. (1976). Inequalities for Stochastic Processes: How to Gamble if You Must, 2nd edn. Dover, New York.

[3] Maitra, A. P. and Sudderth, W. D. (1996). Discrete Gambling and Stochastic Games. Springer, New York.

[4] Pontiggia, L. (2005). Two-person red-and-black with bet-dependent win probabilities. Adv. Appl. Prob. 37, 75-89.

[5] Ross, S. M. (1974). Dynamic programming and gambling models. Adv. Appl. Prob. 6, 598-606. 